\title{
Negative differential resistance in molecular junctions: The effect of the electrodes electronic structure
}

\author{
Natalya A. Zimbovskaya ${ }^{1,2,3}$ and Mark R. Pederson ${ }^{3}$ \\ ${ }^{1}$ Department of Physics and Electronics, University of Puerto Rico-Humacao, CUH Station, Humacao, PR 00791, \\ ${ }^{2}$ Institute for Functional Nanomaterials, University of Puerto Rico, San Juan, PR 00931, and \\ ${ }^{3}$ code 6390, Naval Research Laboratory, 4555 Overlook Ave SW, Washington, DC 20375
}

\begin{abstract}
We have carried out calculations of electron transport through a metal-molecule-metal junction with metal nanoclusters taking the part of electrodes. We show that negative differential resistance peaks could appear in the current-voltage curves. The peaks arise due to narrow features in the electron density of states of the metal clusters. The proposed analysis is based on the ab initio computations of the relevant wave functions and energies within the framework of the density functional theory using NRLMOL software package.
\end{abstract}

PACS numbers: 73.63.Rt, 73.23.Ad, 31.15.A-

Electron transport through molecular-scale systems has been intensively studied in the past two decades [1]. Largely, the unceasing efforts of the research community to further advance these studies are due to important application potentials of single molecules as active elements in various nanodevices intended to complement current silicon based electronics [2]. Among various important properties of the electron transport through metalmolecule junctions one may separate out the negative differential resistance (NDR), that is the decrease of the current $I$ while the bias voltage $V$ across the molecule increases. The NDR efffect was originally observed in tunneling semiconducting diodes [3, 4]. Later, the NDR was viewed in quantum dots [5, 6] and metal-molecule-metal junctions (see e.g. Refs. [6, 7, ,8, 9, 10, 11, 12, 13, 14]).

Several possible scenarios are proposed to explain the NDR occurence in the electron transport through molecules. The NDR could appear due to alignment and subsequent misalignment of the Fermi levels of the leads with molecular orbitals which happens as the bias voltage increases [11, 15]. This may noticeably modify the coupling of the molecule to the leads. The variations in the coupling strengths could serve as an immediate reason for the NDR peaks to appear. Also, such peaks could occur as a Coulomb blockade induced effect [5, 16], and/or they could originate from conformational changes in the molecule [17] and electron interaction with the molecule vibrational modes [7, 18]. It is likely that different mechanisms could play a major part in the NDR appearance in different molecular junctions where it was observed so far. However, reviewing the available experimental data, one may conclude that the most distinguished NDR features in the current-voltage curves (sharp and narrow peaks separated by intervals of extremely low conductivity, like those reported in the Ref. [8]) are usually attributed to the matching-mismatching of the molecule energy levels with those of the leads. Keeping in mind that precisely such NDR characteristics are potentially valuable for molecular electronics applications, we further concentrate on this mechanism.

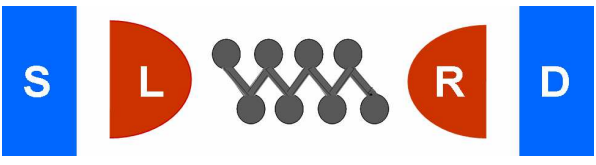

FIG. 1: (Color online) Schematic of the considered system including large electron reservoirs labelled $S$ and $D$, respectively; metal nanoclusters $L$ and $R$; and the molecule placed in between.

Commonly, while studying electron transport through molecules, one assumes that electrodes are large enough to have a feartureless electron density of states below the Fermi energy. In the present work we analyze the NDR effect in the electron transport through a molecular junction where the leads are small metal clusters whose electron density of states reveals sharp and distinct features. This system provides better opportunities to analyze the effects of matching-mismatching of the molecule energy levels with those of the "nanoleads" in the electron transport characteristics. We show that under certain conditions such junctions may show very distinguished multiple NDR features in the $I-V$ characteristics. This demonstrates their potential usefulness in nanoelectronics applications.

To simplify the computational procedure we omit from the present consideration effects originating from Coulomb interactions of electrons and from molecule vibrations. To maintain a steady supply of electrons tunneling through the junction, we assume that the metal clusters ("nanoleads") keep contact with large source and drain electron reservoirs as sketched in the Fig. 1. The latter, however, are separated from the molecule is such a way that electrons cannot directly tunnel from these reservoirs to the molecule. In the absence of the applied bias voltage the whole system is supposed to be in the equilibrium state characterized with the equilibrium Fermi energy $E_{F}$. We write the effective Hamiltonian for the molecule $\left(H_{e f f}\right)$ in the usual form [1, 19, 20, 21]: 


$$
H_{e f f}=H_{M}+H_{L}+H_{R}
$$

where the term $H_{M}$ corresponds to the molecule itself, and $H_{L, R}$ describe the coupling of the latter to the metal nanoclusters.

Omitting for a while the molecule coupling to the leads, one may introduce the retarded Green's function for the molecule. The latter is defined by the matrix equation:

$$
\left[(E+i \eta) \hat{S}-\hat{H}_{M}\right] \hat{G}_{0}^{R}=\hat{I}
$$

where $I$ is the identity matrix, $\hat{S}$ is the overlap matrix,

$$
S_{i j}=\int \psi_{i}^{*}(\mathbf{r}) \psi_{j}(\mathbf{r}) d^{3} r
$$

$\eta$ is an infinitesimal positive parameter $\left(\eta \rightarrow 0^{+}\right), E$ is the energy, and $\psi_{i}, \psi_{j}$ are the orbitals included into the basis set.

Then we employ the Dyson equation. This equation relates the Green's function of the molecule coupled to the leads $\hat{G}^{R}(E)$ to the Green's function of the single molecule $\hat{G}_{0}^{R}(E)$. It reads [22]:

$$
\hat{G}^{R}(E)=\left\{\left[\hat{G}_{0}^{R}(E)\right]^{-1}-\hat{\Sigma}(E)\right\}^{-1} .
$$

Here, the self-energy correction $\hat{\Sigma}(E)$ consists of two terms describing the effect of two clusters:

$$
\hat{\Sigma}(E)=\hat{\Sigma}_{L}(E)+\hat{\Sigma}_{R}(E) .
$$

For convenience, one may introduce a notation $\left[\hat{G}_{0}^{R}(E)\right]^{-1}=\hat{A}(E)$, which allows to simplify the form of the Eq. 4 namely:

$$
\hat{G}^{R}(E)=[\hat{A}(E)-\hat{\Sigma}(E)]^{-1} .
$$

Now, one must calculate matrix elements $A_{i j}(E)$ and $\Sigma_{i j}(E)$ Assuming that the wavefunctions are orthonormalized molecular orbitals, the matrix $\hat{A}(E)$ is a diagonal matrix:

$$
A_{i j}(E)=\left(E+i \eta-E_{i}\right) \delta_{i j}
$$

where $E_{i}$ are the energy eigenstates of the molecule.

The matrix elements of self-energy corrections have the form [1]:

$$
\left(\Sigma_{\beta}\right)_{i, j}=\sum_{k} \frac{W_{i k, \beta}^{*} W_{k j, \beta}}{E-\epsilon_{k, \beta}-\sigma_{k, \beta}} .
$$

here, $\beta \in L, R, W_{i k, \beta}$ are, respectively, the coupling strengths between " $i$ " -th molecule state and " $k$ " -th state on the left/right metallic cluster (lead), $\epsilon_{k, \beta}$ are energy levels of the corresponding leads, and the parameters $\sigma_{k, \beta}$ are the self-energy corrections which originate from the coupling of the clusters to the large electron reservoirs. Their imaginary parts characterize the width of the clusters energy levels. The summation over " $k$ " in the Eq. 8 is carried out over the states of the left/right cluster.

When the bias voltage $V$ is applied across the system shown in the Fig. 1, this causes charge redistribution, and subsequent changes in the energies $E_{i}$ and $\epsilon_{k, \beta}$. In consequence, the matrix elements $A_{i j}$ and $\Sigma_{i j}$ values vary as $V$ changes. This affects the electron transmission function $T$ given by the expression:

$$
T=\operatorname{Tr}\left\{\hat{\Gamma}^{L} \hat{G}^{R} \hat{\Gamma}^{R} \hat{G}^{A}\right\}
$$

where $\hat{\Gamma}^{L, R}=-2 \operatorname{Im} \hat{\Sigma}_{L, R}$, and $G^{A}$ is the advanced Green's function of the molecule $\left(\hat{G}^{A}=\left(G^{R}\right)^{\dagger}\right)$. When the voltage $V$ is applied, the electron transmission which determines transport properties of the molecular junction may significantly depend on its value and polarity.

The electron tunnel current through the junction could be written in the form:

$$
I=\frac{e}{\pi \hbar} \int_{-\infty}^{\infty} d E T\left(E, V_{m o l}\right)\left[f\left(E-\mu_{S}\right)-f\left(E-\mu_{D}\right)\right]
$$

Here, $T\left(E, V_{m o l}\right)$ is the electron transmission, given by the Eq. 9, $f(E)$ is the Fermi distribution function for the energy $E$. Chemical potentials $\mu_{S, D}$ are attributed to the source and drain reservoirs, respectively. They are shifted with respect to the equilibrium Fermi energy due to the applied voltage:

$$
\mu_{S}=E_{F}+(1-\nu) e V ; \quad \mu_{D}=E_{F}+\nu e V
$$

where $e$ is the electron charge and $\nu$ is the division parameter.

We remark that the transmission $T$ in the Eq. 10 actually depends on the voltage applied across the junction $\left(V_{m o l}\right)$. The latter may noticeably differ from the external voltage $V$. The difference originates from both charge redistribution inside the molecule and the electrostatic potential drops between the source/drain reservoirs and the metal clusters. The current-voltage characteristics shapes crucially depend on the electrostatic potential profile in the considered system. If the voltage mostly drops between the large electron reservoirs and the small metal nanoparticles included in the junction $\left(V_{m o l} \ll V\right)$ one may approximate $T(E, V)$ as $T(E, 0)$. Then the applied bias voltage does not change relative positions of the energy levels $\epsilon_{k, \beta}$ and $E_{i}$, and low-temperature characteristics should display step-like shapes. These are typical for electron tunneling through molecules (see e.g. [19, 23]). Current increases as the voltage $V$ increases, and the NDR does not appear. On the contrary, when no significant voltage drop occures between the electron reservoirs and the metal nanoclusters $\left(V_{\text {mol }} \sim V\right)$, the effect of the bias voltage on the energy levels of the clusters $\epsilon_{k, \beta}$ could considerably differ from its effect on the 

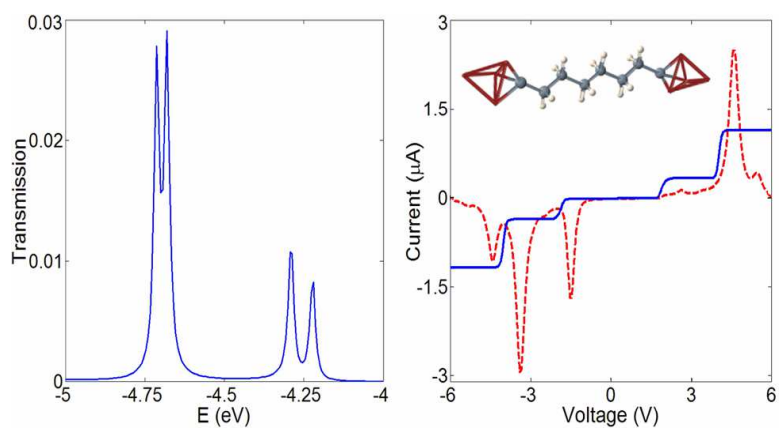

FIG. 2: (Color online) Left panel: The calculated electron transmission function through the molecule $T(E, 0)$ within a certain range of energies $E$. Right panel: The calculated current voltage characteristics for the electron tunneling through the junction. The curves are plotted asuming $T=50 \mathrm{~K}$, and $V_{m o l}=0.1 \mathrm{~V}$ (solid line) and $V_{m o l}=0.9 \mathrm{~V}$ (dashed line).

molecular energy $E_{i}$. Due to these differencies in the voltage induced shifts, the relative positions of the energy levels on the clusters and these on the molecule vary as the voltage changes. This creates opportunities for alignment/disalignment of the molecule orbitals with those associated with the metal clusters. Therefore, in such a case one may expect the NDR to occur.

To confirm the above suppositions we carried out calculations of the tunnel electric current through a junction which consists of two copper nanoclusters and an alaphatic-saturated hydrocarbon chain situated in between them (see Fig. 2). The relevant eigenenergies and matrix elements included in the Eqs. 33 are computed using the NRLMOL software package [24]. First we performed full self-consistent calculations on the considered nanosystem. In addition to solving the KohnSham equations self consistently we have optimized the geometry as well. Then we use Lowden's method [25] of symmetrical orthogonormalization to construct atom centered wannier-like functions [26] from the nonorthogonal atom-centered gaussian orbitals. We reconstructed the Hamiltonian matrix in this basis and block diagonalized it separating out the blocks coprresponding to the hydrocarbon chain and the copper clusters. Small offdiagonal elements between the L-M and M-R blocks of the Hamiltonian describe the coupling of the molecule chain to the nanoleads.

The final matrix was used to determine the various matrices needed in Eq. 1,[11, In the following calculations of the tunnel current we assumed $V_{\text {mol }}=0.1 \mathrm{~V}$ and $V_{m o l}=0.9 \mathrm{~V}$, respectively. The resulting $I-V$ curves are presented in the Fig. 2. One can see that when the electrostatic potential mostly drops between the sourse/drain reservoirs and the nearby copper clusters $\left(V_{m o l}=0.1 V\right)$, the corresponding $I-V$ curve reveals a stepwise profile without NDR features. On the contrary, distinct NDR peaks emerge provided that there exists a significant drop in the electrostatic potential between the copper clusters and the molecule tips $\left(V_{\text {mol }}=0.9 \mathrm{~V}\right)$. Also, one may notice that some NDR peaks are rather sharp and narrow, and the current peak values are much greater than in the valleys between the peaks. This is consistent with the experimental data reported in the [8].

In conclusion, we have considered a metal-moleculemetal tunneling junction where metal leads are nanoparticles. Due to the extremely small size of these metal clusters their electron density of states reveals distinct features which are washed out for larger electrodes. We did show that NDR peaks could appear due to alignment and subsequent disalignment of the energy levels of the metal nanoclusters with those of the molecule when the voltage applied across the junction varies. The proposed analysis is based on simple assumptions concerning the electrostatic potential distribution inside the junction. It seems unlikely that these simplifications could cause qualitative distortions in the considered NDR manifistations. However, to quantitatively analyze the effect one must properly compute the electrostatic potential profile employing a self-consistent computational procedure. Also, one should take into account the effects of molecular vibrations which could result in extra NDR features superimposed upon those presently analyzed. Nevertheless, we do believe that we showed that the tunneling junctions including a molecule placed in between metallic nanoparticles could exhibit distinct NDR peaks in the $I-V$ curves. This makes such junctions useful is designing nanoelectronic devices.

Acknowledgments: We thank G. M. Zimbovsky for help with the manuscript. NZ acknowledges support from the ASEE and ONR Summer Faculty Research Program.

[1] S. Datta, Quantum Transport: Atom to Transistor (Cambridge University Press, 2006).

[2] See e.g. G. Cuniberti et al (Ed), Introduction to Molecular Electronics (Springer, Berlin, 2005).

[3] L. Ezaki and P. J. Stiles, Phys. Rev. Lett. 6, 1108 (1966).

[4] L. L. Chang, E. E. Mendez, and C. Tejedor, Resonant Tunneling in Semiconductors (Plenum, New York, 1991).

[5] M. A. Kastner, Rev. Mod. Phys. 64, 849 (1992).

[6] L. W. Yu, K. J. Chen, J. Song. J. M. Wang, J. Xu. W. Li, and X. F. Huang, Thin Solid Films 515, 5466 (2007).

[7] J. Gandioso, L. J. Lauhon, and W. Ho. Phys. Rev. Lett. 85, 1918, (2000).

[8] F.-R. F. Fan, R. Y. Lai, J. Cornil, Y. Karzari, J-L. Bredas, L.-T. Cai, L. Cheng, Y. Yao, D. W. Price, Jr., S. M. Dirk, J. M. Tour, and A. J. Bard, J. Am. Chem. Soc. 126, 2568 (2004).

[9] A. Salomon, R. Arad-Yellin, A. Shanzer, A. Karton, and D. Cahen, J. Am. Chem. Soc. 126, 11648 (2004).

[10] M. Grobis, A. Wachowiak, R. Yamachica, and M. F. 
Crommie, Appl. Phys. Lett. 86, 204102 (2005).

[11] J. Repp, G. Meyer, S. M. Stojkovic, A. Gourdon, and C. Joachim, Phys. Rev. Lett. 94, 026803 (2005).

[12] J. J. Davis, T. Wang, A. Morgan, G. Zhang, J. Zhao, Faraday Discuss. 131, 167 (2006).

[13] E. D. Mentovich, I. Kalifa, A. Tsukernik, A. Caster, N. Rosenberg-Shraga, H. Marom, M. Gozin, S. Richter, Small 4, 55 (2007).

[14] G. Maruccio, P. Marzo, R. Krahne, A. Passaseo, R. Cingolani, and R. Rinaldi, Small 3, 1184 (2007).

[15] Y. Xue, S. Datta, S. Hong, R. Reifenberger, J. I. Henderson, and C. P. Kubiak, Phys. Rev. B 59, R7852 (1999).

[16] N. Simonian, J. Li, and K. Likharev, Nanotechnology 18, 424006 (2007).

[17] V. Mujica, A. Nitzan, S. Datta, M. A. Ratner, and C. P. Kubiak, J. Phys. Chem. B 107, 91 (2003).

[18] M. Yu. Galperin, M. A. Ratner, and A. Nitzan, Nanolet- ters 5, 125 (2005).

[19] N. A. Zimbovskaya and G. Gumbs, Appl. Phys. Lett. 81 1518 (2002).

[20] Y. Xue, S. Datta, and M. A. Ratner, J. Chem. Phys. 115, 4292 (2001).

[21] M. Yu. Galperin, M. A. Ratner, and A. Nitzan, J. Chem. Phys. 121, 11965 (2004).

[22] M. Yu. Galperin, A. Nitzan, and M. A. Ratner, Phys. Rev. Lett. 86, 166803 (2006).

[23] V. Mujica, A. E. Roitberg, and M. A. Ratner, J. Chem. Phys. 112, 6834 (2000).

[24] M. R. Pederson, D. V. Porezag, J. Kortis, and D. C. Patton, Phys. Status Solidi B 217, 197 (2000).

[25] P. O. Lowden, J. Chem Phys. 18, 365 (1950).

[26] M. R. Pederson and C. C. Lin, Phys. Rev. B 35, 2273 (1985). 